\title{
Multidisciplinary Aerodynamic Design of a Rotor Blade for an Optimum Rotor Speed Helicopter
}

\author{
Jiayi Xie, Zhifeng Xie, Ming Zhou and Jun Qiu* \\ Institute for Aero-Engine, School of Aerospace Engineering, Tsinghua University, Tsinghua Yuan No. 1, \\ Beijing 100084, China; xiejy12@mails.tsinghua.edu.cn (J.X.); xzhf@tsinghua.edu.cn (Z.X.); \\ zmzlh@mail.tsinghua.edu.cn (M.Z.) \\ * Correspondence: qiujun07@tsinghua.org.cn; Tel.: +86-10-6279-4843 \\ Academic Editor: Kuang-Chao Fan \\ Received: 16 May 2017; Accepted: 13 June 2017; Published: 20 June 2017
}

Featured Application: This work is potential to apply in the design of optimum rotor speed and long-endurance helicopter.

\begin{abstract}
The aerodynamic design of rotor blades is challenging, and is crucial for the development of helicopter technology. Previous aerodynamic optimizations that focused only on limited design points find it difficult to balance flight performance across the entire flight envelope. This study develops a global optimum envelope (GOE) method for determining blade parameters-blade twist, taper ratio, tip sweep-for optimum rotor speed helicopters (ORS-helicopters), balancing performance improvements in hover and various freestream velocities. The GOE method implements aerodynamic blade design by a bi-level optimization, composed of a global optimization step and a secondary optimization step. Power loss as a measure of rotor performance is chosen as the objective function, referred to as direct power loss (DPL) in this study. A rotorcraft comprehensive code for trim simulation with a prescribed wake method is developed. With the application of the GOE method, a DPL reduction of as high as $16.7 \%$ can be achieved in hover, and $24 \%$ at high freestream velocity.
\end{abstract}

Keywords: ORS-helicopter; blade aerodynamic design; global optimum envelope (GOE); prescribed wake method

\section{Introduction}

Aerodynamic design of helicopter rotors is a challenging task, especially when designing it for its optimal performance in both hover and forward flight conditions. There are two primary reasons for the trade-off problem between hover and forward flight: (1) the major two causes of rotor power loss-induced power loss and profile power loss-have different characteristics in different flight conditions. In hover and low advance ratio flight, the induced power loss dominates; while profile power loss rises with flight speed increases, and gradually becomes the major part; and (2) some rotor parameters have opposite effects on the two forms of power loss. For example, a large disk area benefits hover FM (figure of merit), but incurs a performance penalty in forward flight. The blade needs to have a large negative value for improving hover FM, while a large negative twist may cause forward flight performance to deteriorate [1]. It is also known that a reduced rotor speed is advantageous in reducing rotor power requirements at moderate flight speeds, while a large rotor speed is necessary to maintain a high take-off capacity for the helicopter.

In recent years, many research studies have been published that solve such trade-off. Le Pape [2] implemented three optimization algorithms in the 7A rotor and ERATO (Etude d'un Rotor Aéroacoustique Technologiquement Optimisé) rotor aerodynamic designs. Constraint methods and weighting of objective functions (WOF) were applied to hover FM and forward flight rotor shaft torque 
balance. A genetic algorithm was implemented to optimize the ERATO rotor in three points: hover, 0.2 advance, and 0.4 advance. Léon et al. [3] adopted the Nash game method for the ERATO blade to optimize blade planform, twist and chord distribution. A bi-objective strategy was composed, with hover FM maximization and rotor power minimization in a 0.334 advance ratio. The power was shown to decrease by $1 \%$ and the hover FM was shown to increase by $2 \%$ in the last Nash equilibrium point. A WOF (weighting of objective functions) method to consider both hover and forward flight performances was carried out by Imiela [4], and weight analysis was done at a 0.4 advance ratio. The contribution of this study was to couple Computational Fluid Dynamics (CFD) and Computational Structural Dynamics (CSD) methods, providing a high-fidelity optimization framework. However, it should be noted that applying high-fidelity modeling methods to the optimization of an entire envelope leads to an expensive time-consumption problem. Leusink et al. [5] carried out a multi-fidelity optimization. The low-fidelity model was utilized to reduce parameter space, and the high-fidelity surrogate model from CFD results was implemented in the optimization procedure. The set of Pareto optimal solutions with respect to hover and flight performances was found out through genetic algorithm. Bailly et al. [6] recently studied the effects of blade twist and tip anhedral on required power. They demonstrated that the induced power loss could be reduced by $18.4 \%$ with an optimal blade twist, and by $18.8 \%$ with an optimal blade twist and anhedral.

Variable blade technology has been a focus over recent decades. It has been demonstrated to be a promising way of improving rotor performance $[7,8]$. In general, variable blade technology can be classified into variable blade geometry and variable rotor speed. The variable-speed rotor adopts a transmission system or wide speed range engine to change revolution, ensuring that operation is optimal in different conditions. Reduced rotor speed is advantageous to improving hover FM, saving energy in forward flight and extending the helicopter flight envelope [9,10]. Boeing applied an optimum rotor system (ORS) to unmanned aerial vehicles (UAV) A160/ A160T in the last century, which achieved continuous flight for $18.7 \mathrm{~h}$ in 2008 . The maximum flight speed reached up to $263 \mathrm{~km} / \mathrm{h}$ in a real flight test. Guo [11] modeled UH-60A; and optimized rotor speed at different forward velocities, rotor required power, and torque were investigated in this study. A power reduction of up to $15 \%$ was confirmed with the optimal rotor speed, while a $14 \%$ increase of shaft torque was obtained. Garavello and Benini [12] combined the UH-60A and GE-T700 models to reveal benefits and drawbacks of a variable-speed helicopter, and found that a wide speed range turboshaft engine may be required in order to save energy in an ORS-helicopter. Han and Barakos [13] examined the benefits from variable tail rotor speed, which concluded the fact that optimal tail rotor speed could achieve greater power saving in cruise than in hover, and a high advance ratio. The maximum power reduction was up to $30 \%$ of the baseline tail rotor.

Variable blade geometry usually includes variable span, blade twist and chord extension. Because variable blade geometry allows the rotor to change to an optimal state based on flight conditions, it is the preferred way of balancing hover and forward flight performances. Khoshlahjeh and Gandhi [7] examined the benefits attained through chord extension morphing and variable rotor speed in a Black Hawk UH-60A helicopter. It was found that the required power could be reduced by $19.5 \%$ at sea level with $16,000 \mathrm{lbs}$ gross weight, and by $13.4 \%$ at sea level with $183,000 \mathrm{lbs}$, with rotor speed set to $85 \%$ of the baseline. Ramanujam and Abhishek [14] applied a particle swarm optimization for the UH-60A rotor blade, improving power consumption by employing variable radius, chord, twist, revolution and trailing-edge plate extension.

The main purpose of this study is to determine the optimal blade parameters in an ORS-helicopter through an aerodynamic design considering hover FM and forward flight performance simultaneously. The design variables include blade twist, taper ratio, blade tip sweep, and rotor speed. The quasi-static solutions of flap motion and prescribed wake are adopted in this study. The main contribution of this study is to establish a novel design method called Global Optimum Envelope (GEO) for blade optimization across the entire flight condition. The proposed GOE method integrates with a comprehensive helicopter code developed in-house, making the design procedure more efficient. 
It separates aerodynamic design into a global optimization step, allowing parameters to change with freestream velocities; and a secondary-optimization step, searching for the solution closest to the global optimization: First, a multi-variable hypersurface representing the relationships among objective, blade parameters and freestream velocities is established, and section lines of the objective corresponding to different rotor speeds are found. Second, the minimal objective value is obtained by rotor speed optimization at each of the given blade parameters. Finally, a global optimized objective with freestream velocity is identified at the envelope, with all blade parameters included; and the parameters with optimal rotor speed are determined as those optimal for the ORS-helicopter by minimizing the deviation between objective values and global optimized values with respect to freestream velocities. Another contribution of this study is to derive rotor power loss based on blade element theory, picking out the parts related to blade parameters and forming an objective function in rotor optimization. The effects of blade variations on rotor performance are investigated in this study. A multi-variable optimization is implemented for overall freestream velocities. The results of the GOE method and its comparison with variable blade technology are given.

\section{Methodology}

\subsection{Framework}

The study proposes a three-step design method-Global Optimum Envelope (GOE)—-to determine blade parameters for an ORS-helicopter, in order to reduce rotor power loss $P_{\text {rot }}$ and balance hover FM with forward flight performance. Figure 1 shows the framework of the GOE method, where $\mathbf{X}$ expresses blade design parameters, $\omega$ is rotor revolution, and $V_{f}$ is freestream velocity. In the first step, the relationship among objective, blade parameters, rotor speed and freestream velocity is established. In the second step, the objective function is minimized at given blade parameter sets. In the last step, the global optimal solution is found with variable blade geometry and rotor speed, and the GOE optimal solution is achieved at frozen blade parameters. The objective function, $P_{\text {rot }}$, of the GOE method is obtained through isolated rotor trim in different parameters, rotor speeds and freestream velocities. The rotor trim is implemented by an in-house developed rotorcraft comprehensive code, based on flap modeling and a prescribed wake method in this study. Quasi-steady solutions for flap coefficients are obtained by the Newton method with Steffensen acceleration in this study. The induced velocity around the blade section is computed by the prescribed wake method. An iteration between blade flap motion and induced velocity computation is completed when induced velocity is converged. The aerodynamic forces of blade sections are computed based on the blade elements in this study, through interpolation of the aerodynamic coefficients C 81 table.

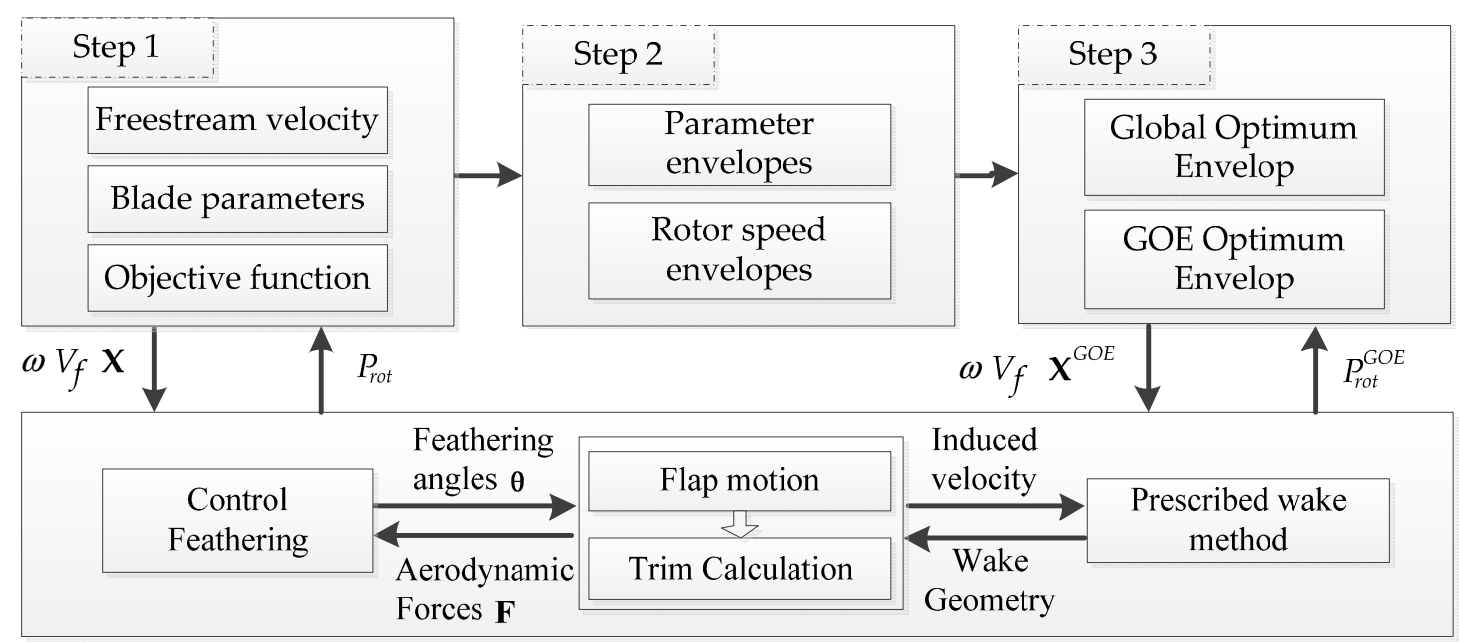

Figure 1. Framework of GOE method to determine blade parameters. 


\subsection{Modeling}

\subsubsection{Forward Flight Trim}

Affected by blade flap motion and rotor speed, the air velocity around a 2-D airfoil can be expressed by

$$
\left(\begin{array}{l}
u_{r} \\
u_{t} \\
u_{p}
\end{array}\right)=\left(\begin{array}{ccc}
\cos \beta & 0 & \sin \beta \\
0 & 1 & 0 \\
-\sin \beta & 0 & \cos \beta
\end{array}\right)\left(\begin{array}{ccc}
\cos \psi & \sin \psi & 0 \\
-\sin \psi & \cos \psi & 0 \\
0 & 0 & 1
\end{array}\right)\left(\begin{array}{c}
-\mu_{h x} \\
-\mu_{h y} \\
\lambda_{h}
\end{array}\right) \omega R+\left(\begin{array}{c}
0 \\
\omega \widetilde{r} \\
\dot{\beta}(r-\varepsilon R)
\end{array}\right)
$$

where $\widetilde{r}=\varepsilon R+(r-\varepsilon R) \cos \beta$.

The blade section aerodynamic forces can be formulated by airfoil aerodynamic coefficients, air dynamic pressure and the section chord.

$$
\left\{\begin{aligned}
d L & =\frac{1}{2} \rho u_{a}^{2} C_{L} c d r \\
d D & =\frac{1}{2} \rho u_{a}^{2} C_{D} c d r
\end{aligned}\right.
$$

where section lift $d L$ is normal to inflow direction locally, and section drag $d D$ is parallel to the inflow direction. The airfoil lift coefficient and drag coefficient are both interpolated by the C81 table.

Flap motion is modeled by moment balance at the flap hinge.

$$
M_{a}=-\left(M_{c}+M_{i}+M_{k}\right)
$$

where $M_{c}=-\omega^{2}\left(I_{\beta} \cos \beta+\varepsilon R M_{\beta}\right) \sin \beta$, representing centrifugal moment; $M_{i}=-I_{\beta} \ddot{\beta}$, representing flap inertia moment; $M_{k}=-K_{\beta} \beta$, expressing restrain moment at the hinge. Aerodynamic moment $M_{a}$ is expressed by the integral of $d F_{z}$ along the blade span.

$$
M_{a}=\int_{\varepsilon}^{1} R d F_{z}=R \int_{\varepsilon}^{1} \cos \phi d L-\sin \phi d D
$$

In the hypothesis of quasi-steady motion, the flap can be solved as

$$
\beta=\beta_{0}+\beta_{c} \cos \psi+\beta_{s} \sin \psi
$$

This study adopts Beddoes' prescribed wake method to model tip vortex, and calculates induced velocity. The position of the certain vortex is expressed following Kutta condition [15].

$$
\left(\frac{\partial \mathbf{r}}{\partial \psi_{b}}+\frac{\partial \mathbf{r}}{\partial \psi_{t}}\right)=\boldsymbol{\mu}+\lambda(\mathbf{r}, t)
$$

where the vortex position $\mathbf{r}\left(\psi_{b}, \psi_{t}\right)$ can be parameterized by present blade azimuth $\psi_{b}$ and shed azimuth $\psi_{t}$; the freestream velocity vector is expressed by $\mu$; and the induced velocity vector is expressed by $\lambda$. The $Z$-axis component of the vortex position can be expressed as

$$
z_{v}=-\mu_{z}\left(\psi_{b}-\psi_{t}\right)+\int_{\psi_{t}}^{\psi_{b}} \lambda_{i} d \psi
$$

Linear inflow distribution is assumed by Beddoes. Induced velocity within the rotor disc can be expressed as

$$
\lambda_{i}(x, y)=-\lambda_{i o}\left[1+E\left(\frac{8}{15 \pi}+x-|y|^{3}\right)-2 \mu_{x} y\right]
$$


Induced velocity in the far field

$$
\lambda_{i}(x, y)=-2 \lambda_{i o}\left[1+E\left(\frac{8}{15 \pi}-|y|^{3}\right)-2 \mu_{x} y\right]
$$

where $|y|^{3}$ reflects vortex roll-up near the blade tip, and $-2 \mu_{y}$, from Drees [16], represents lateral variation. The constant term $8 / 15 \pi$ is introduced by van der Wall [17] , in agreement with momentum theory. Beddoes applied $E=\chi$ to represent longitudinal non-uniformity, while $E=\chi / 2$ is adopted in this paper, instead, which was found to be better in agreement with experiments and the free vortex wake method [15], where $\chi=\arctan \left(\frac{\mu_{x}}{\mu_{y}+\lambda}\right)$.

The $x$ and $y$ components of vortex position can be expressed as

$$
\left\{\begin{array}{c}
x_{v}=r_{v} \cos \psi_{t}+\mu_{x}\left(\psi_{b}-\psi_{t}\right) \\
y_{v}=r_{v} \sin \psi_{t}
\end{array}\right.
$$

where $r_{v}$ is the radius station vortex shed.

We discretized the wake filament by straight-line elements, and chose the endpoints as the control points as shown in Figure 2. Therefore, the induced velocity by certain vortex element can be expressed by the core-modification Biot-Savart law

$$
d v_{i}=\frac{\Gamma}{4 \pi}\left[\frac{h^{2}}{\left(r_{c}^{4}+h^{4}\right)^{1 / 2}}\right] \mathbf{r}_{1} \times \mathbf{r}_{2}\left(\frac{1}{r_{1}}+\frac{1}{r_{2}}\right) \frac{1}{r_{1} r_{2}+\mathbf{r}_{1} \cdot \mathbf{r}_{2}}
$$

where $\mathbf{r}_{1}, \mathbf{r}_{2}$ express the position vectors from vortex control points to the calculating point, and $h$ expresses the distance between the vortex element and the calculation point. The core radius $r_{c}$ for the tip vortex is set to $10 \%$ of the chord length. The induced velocity in the certain point $\mathrm{P}$ is the integral of all trail vortices in the wake at the moment.

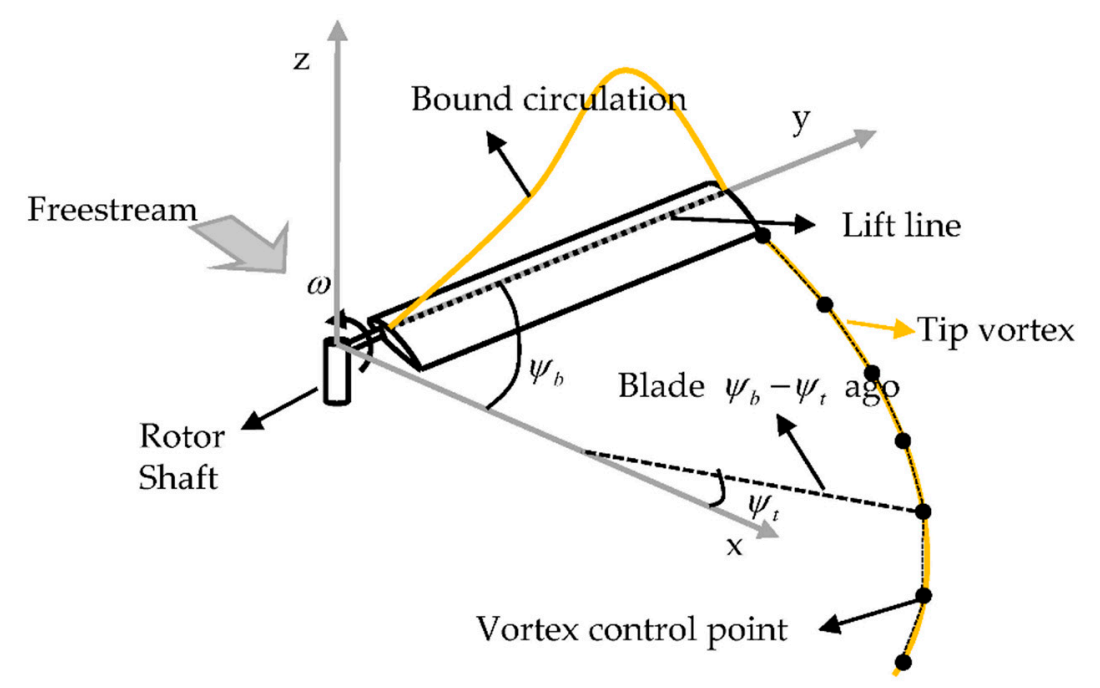

Figure 2. Definition of rotor tip wake geometry.

This study applies the lift-line model to represent the blade, which is set at one-quarter of the chord and along the blade span. The calculation points for induced velocity are set at one-quarter of the chord. Based on the Kutta-Joukowsky theorem, bound circulation and blade section lift have a relationship that can be expressed as

$$
\Gamma_{b}=\frac{1}{2} u_{a} c C_{L}
$$


The trail vortex strength can be expressed as a variation of bound circulation,

$$
\Gamma(\psi, r)=\Gamma_{b}(\psi, r)-\Gamma_{b}(\psi, r+d r)
$$

The helicopter rotor is manipulated through blade feathering, achieving TPP (Tip-path plane) tilt and providing hub forces and moments to maintain the vehicle's equilibrium in flight. The isolated rotor trim equations can be expressed as in Equation (14). This study adopts the Newton-Raphson method to solve the trim equations.

$$
\left(\begin{array}{c}
\theta_{0} \\
\theta_{c} \\
\theta_{s}
\end{array}\right)=\left(\begin{array}{lll}
\frac{\partial C_{T}}{\partial \theta_{0}} & \frac{\partial C_{T}}{\partial \theta_{c}} & \frac{\partial C_{T}}{\partial \theta_{s}} \\
\frac{\partial \beta_{c}}{\partial \theta_{0}} & \frac{\partial \beta_{c}}{\partial \theta_{c}} & \frac{\partial \beta_{c}}{\partial \theta_{s}} \\
\frac{\partial \beta_{s}}{\partial \theta_{0}} & \frac{\partial \beta_{s}}{\partial \theta_{c}} & \frac{\partial \beta_{s}}{\partial \theta_{s}}
\end{array}\right)^{-1}\left(\begin{array}{c}
C_{T}-C_{\text {target }} \\
\beta_{c} \\
\beta_{s}
\end{array}\right)
$$

\subsubsection{Rotor Direct Power Loss}

The rotor power loss is derived in order to determine an objective function in this study. The incremental torque can be expressed as

$$
d Q=\widetilde{r} d F_{x}
$$

where $d F_{x}$ is blade section backward force.

Combine Equation (2) and Equation (15), and represent $d Q$ as two parts:

$$
d Q=\widetilde{r} \tan \varphi d F_{z}+\widetilde{r} \frac{d D}{\cos \varphi}=d Q_{i}+d Q_{o}
$$

where $d F_{z}$ is blade section normal force; $d Q_{i}$ reflects the cost to provide lift perpendicular to the blade section; $d Q_{o}$ reflects the cost to rotate the blade in a viscous fluid.

Operate the rotor backward force $d H$ in a similar way

$$
d H=(\tan \varphi \sin \psi-\sin \beta \cos \psi) d F_{z}+\frac{\sin \psi}{\cos \varphi} d D=d H_{i}+d H_{o}
$$

Since there is no y-direction quantity in a hub wind coordinate, the quantities are rearranged with respect to hub wind direction, and the quantity $R \mu d H_{i}+d Q_{i}$ as $d Q_{1}$ is defined as

$$
d Q_{1}=(R \mu \sin \psi+\widetilde{r}) \tan \varphi d F_{z}-R \mu \sin \beta \cos \psi d F_{z}
$$

Combine Equation (1), and note that $\tan \varphi=u_{p} / u_{t}$,

$$
d Q_{1}=\frac{\dot{\beta}}{\omega}(r-\varepsilon R) d F_{z}+\lambda_{h} R \cos \beta d F_{z}
$$

The integral within one revolution of first part in Equation (20) must be zero, since the $d F_{z}$ and flap angle $\beta$ are periodic in a steady trim.

Rewrite $d Q$ with the relationship $\lambda_{h}=\lambda_{i}-\mu_{x} \alpha_{f}$ in the hub reference system,

$$
d Q=\lambda_{i} R \cos \beta d F_{z}+\left(R \mu d H_{o}+d Q_{o}\right)-R \mu d H-\mu_{x} \alpha_{f} R \cos \beta d F_{z}+\frac{\dot{\beta}}{\omega}(r-\varepsilon R) d F_{z}
$$

Rotor power loss is expressed as

$$
P=\omega Q=P_{i}+P_{o}+P_{f}
$$


where $P_{i}=\frac{\omega n_{b} R^{2}}{2 \pi} \int_{0}^{2 \pi} \int_{\varepsilon}^{1} \lambda_{i} d T, P_{o}=\frac{\omega n_{b} R^{2}}{2 \pi} \int_{0}^{2 \pi} \int_{\varepsilon}^{1}\left(\mu d H_{o}+\frac{1}{R} d Q_{o}\right), P_{f}=-\frac{\omega n_{b} R^{2}}{2 \pi} \int_{0}^{2 \pi} \int_{\varepsilon}^{1} \mu d H+\mu_{x} \alpha_{f} d T$.

The component $P_{i}$ is defined as induced power, which reflects the power consumption to produce rotor thrust, with the pattern of the dot product of induced velocity and section lift. The component $P_{o}$ is defined as profile power, which reflects the power consumption to rotate the rotor in the air. The component $P_{f}$ is determined by the entire helicopter trim state, fuselage equivalent drag area and gross weight.

Define

$$
P_{\text {rot }}=P_{i}+P_{o}
$$

as the objective function in this study. It can be seen that the term $P_{\text {rot }}$, called direct power loss (DPL), is a component representing rotor design merit and rotor performance. It is also advantageous to adopt DPL as the objective function, since it can represent hover FM $\left(F M=P_{i i d} / P_{r o t}\right)$ and rotor performance in forward flight in one equation.

\subsection{Parameter Determination}

The rotor aerodynamic optimization design is separated into two procedures by the GOE method; the first one is a global optimization procedure, and the second one is a secondary-optimization procedure, with three steps.

Step 1:

$$
\left\{\begin{array}{c}
S_{\omega}\left(P_{r o t}, \mathbf{X}, V_{f}\right)=f_{1}\left(P_{r o t}, \mathbf{X}, V_{f} \mid \omega\right) \\
L_{\omega}\left(P_{r o t}, V_{f}\right)=f_{2}\left(P_{r o t}, V_{f} \mid \mathbf{X}, \omega\right)
\end{array}\right.
$$

Step 2:

$$
\left\{\begin{aligned}
P_{\text {rot }}\left(V_{f} \mid \mathbf{X}\right)=\min _{\omega}\left[L_{\omega}\left(P_{\text {rot }}, V_{f} \mid \mathbf{X}\right)\right] \\
\text { s.t. } \quad \omega=\omega\left(V_{f} \mid \mathbf{X}\right)
\end{aligned}\right.
$$

Step 3:

$$
\begin{gathered}
\left\{\begin{array}{r}
P_{\text {rot }}^{g l}\left(V_{f}\right)=\min _{\mathbf{X}}\left[P_{\text {rot }}\left(V_{f} \mid \mathbf{X}\right)\right] \\
\text { s.t. } \quad \mathbf{X}=\mathbf{X}\left(V_{f}\right)
\end{array}\right. \\
\left\{\begin{array}{c}
\min _{\omega} \int_{V_{f}}\left[P_{\text {rot }}^{g l}\left(V_{f}\right)-P_{\text {rot }}^{G O E}\left(V_{f}\right)\right]^{2} \\
\text { s.t. } \omega=\omega\left(V_{f}\right), \mathbf{X}=\mathbf{X}^{G O E}
\end{array}\right.
\end{gathered}
$$

where $P_{r o t}^{G O E}\left(V_{f}\right)=P_{r o t}\left(V_{f} \mid \mathbf{X}=\mathbf{X}^{G O E}\right) . \quad S_{\omega}\left(P_{r o t}, \mathbf{X}, V_{f}\right)$ represents the relationship among the objective function, design parameters and freestream velocity, corresponding to certain rotor speed. $L_{\omega}\left(P_{r o t}, V_{f} \mid \mathbf{X}\right)$ expresses DPL section lines by crossing hypersurfaces $S_{\omega}\left(P_{r o t}, \mathbf{X}, V_{f}\right)$. The operator $\int_{V_{f}}(\cdot)$ means to consider overall freestream conditions. An optimization package supported by MATLAB $^{\circledR}$ is used in this study. Bound constrained nonlinear optimization is implemented to find the optimal solution $P_{r o t}^{g l}$ for a variable blade, and a nonlinear least-squares with Levenberg-Marquardt algorithm [18] is applied to find the GOE solution.

\section{Validation}

An ultra-light helicopter is used as the baseline for the GOE method in this study. The helicopter parameters are listed in Table 1. 
Table 1. Main parameters of an ultra-light helicopter.

\begin{tabular}{cc}
\hline \multicolumn{2}{c}{ Vehicle Parameters } \\
\hline Gross weight & $414 \mathrm{~kg}$ \\
CG position & $19 \mathrm{~mm}$ in front of hub center; \\
Parasite drag area ratio & $1285 \mathrm{~mm}$ beneath the hub \\
\multicolumn{2}{c}{ Rotor Parameters } \\
\hline Blade number & 0.0315 \\
Rotor type & Teetering; rectangular blade \\
Nominal speed & $525 \mathrm{rpm}$ \\
Rotor diameter & $7010 \mathrm{~mm}$ \\
Chord length & $170 \mathrm{~mm}$ \\
Twist angle & $-8 \mathrm{deg}$ \\
Pre-cone angle & $3 \mathrm{deg}$ \\
Shaft tilt & $3 \mathrm{deg}$ \\
Solidity & 0.0309 \\
Lock number & 5.01 \\
Airfoil & Boeing VR-7 \\
\hline
\end{tabular}

Since there is a lack of wake data, the 2MRTS wake experiment $[19,20]$ is adopted to validate the prescribed wake method (PWM) used in this study. The following conditions are implemented in the validation: thrust coefficient $C_{T}=0.0064$, advance ratio $\mu=0.15$, and rotor shaft $\alpha_{s}=-3^{0}$. The induced velocity is computed on a plane one-chord above TPP, the same as the experiment setting. Time-average induced velocity is shown in Figure 3. Comparing with experimental data (EXP) [19] and free vortex method (FVM) [21], it is well understood that the prescribed wake method (PWM) is able to capture the non-uniform distribution of induced velocity, especially in the blade tip region. Slight deviations can be seen in the root region, which may be due to ignoring the root cut-out effect in this study, where root vortices may exist [20]. The strongest downwash presents near the tip for a rotating blade; hence, non-uniform distribution and rotor performance estimation are a major responsibility in induced velocity.
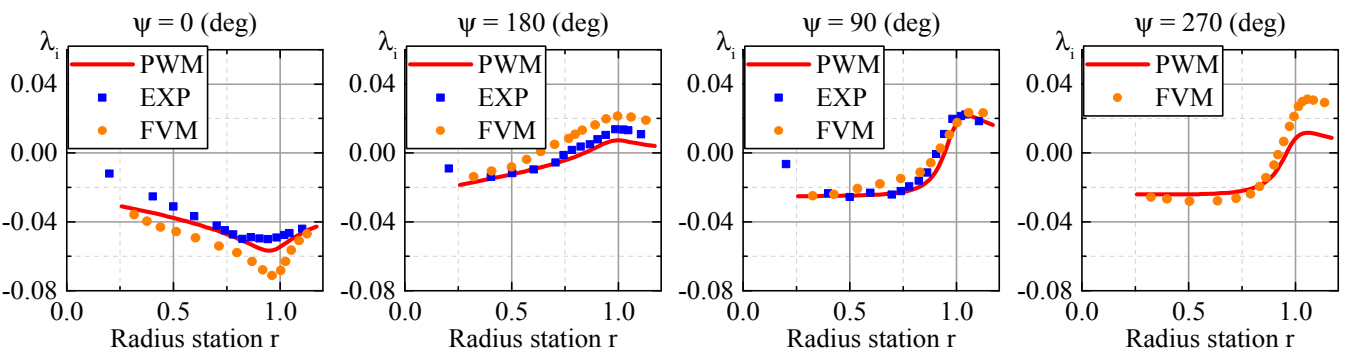

Figure 3. Induced velocity on TPP for the wake measurement experiment. PWM expresses the present prescribed wake method; FVM expresses the free-vortex method referred to Bhagwat [21]; EXP is the experimental data [19].

Figure 4 shows the trim results of the ultra-light helicopter, comparing with UMARC and CAMRAD II results from Shen et al. [22]. With the increase in flight speed, the longitudinal flap coefficient $\beta_{c}$ increases, providing propulsive force through the tilted TPP. The lateral trim results at present have the same trends as UMARC and CAMRAD II. With an increase in flight speed, a steady deviation of about 1 degree between present simulation and CAMRAD II always exists, the same as in the UMARC result. This may be caused by the inflow model, as Shen, Chopra and Johnson [22] have mentioned. With a high advance ratio, the lateral flap coefficient $\beta_{s}$ rises more slowly than UMARC, and may benefit from the prescribed wake method. As for rotor power loss, minimal consumption is 
achieved with the medium advance ratio, due to a reduction of induced power $P_{i}$ and an increase of profile power $P_{o}$ as flight speed rises. With flight speed increases, $P_{i}$ is lower than in hover, which is because there is more mass flux contributed by freestream in forward flight, a bit of induced inflow can produce adequate thrust for the entire helicopter. Therefore, it can be seen that $P_{o}$ is larger than $P_{i}$ in forward flight, and becomes the bottleneck in performance improvement. Figure 5 depicts the time history of $P_{i}$ and $P_{o}$ of a blade. In hover, the time histories of both power losses are steady, because of the symmetric aerodynamic environment; whereas in forward flight, $P_{i}$ reduces from 0-degree azimuth to 180-degree azimuth, and becomes negative when the induced flow is upwash. The larger $P_{o}$ is consumed near the blade tip, especially in the advancing blade when dynamic pressure is highest. Therefore, a tapered or tip swept blade could be implemented to reduce rotor profile power loss in this study.
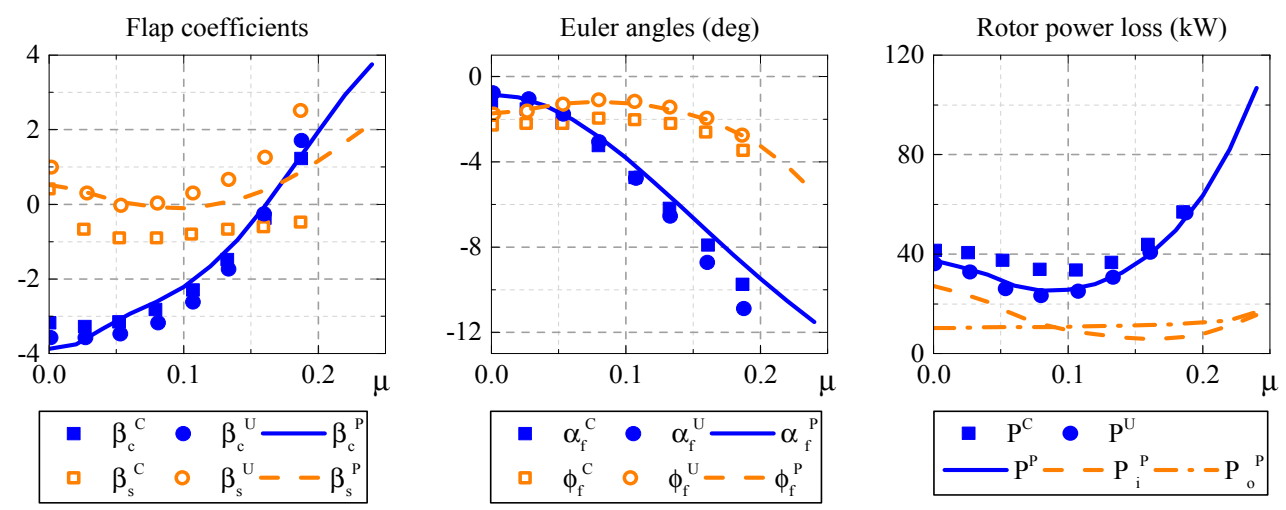

Figure 4. Trim results of certain ultra-light helicopter (The superscript $U$ and $C$ express simulation by UMARC and CAMRAD II , and superscript P expresses present simulation.).

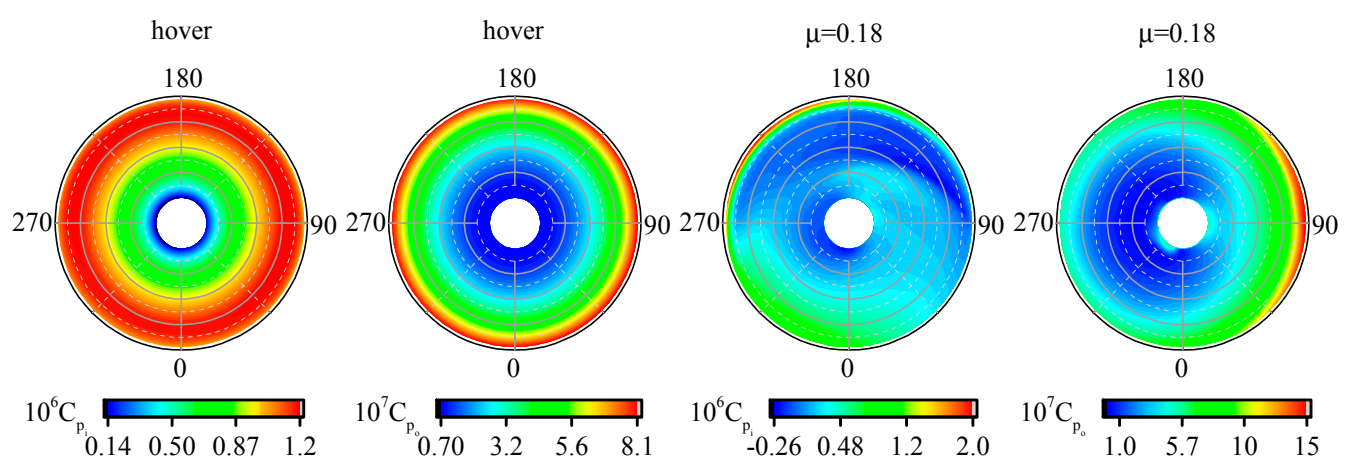

Figure 5. Time history of induced power and profile power for a blade.

\section{Results and Discussion}

Isolated rotor trim is implemented to study blade parameter effects and determination in this study, ensuring the rotor shaft orientation is unchanged, with the whole helicopter trim remaining as given in Figure 4. The main purpose is to simplify the trim routine and accelerate the GOE procedure. Hovering and $\mu=0.18$ forward flight have been chosen to study the effects of blade parameters on DPL, since under these two conditions induced power and profile power, respectively dominate in DPL.

\subsection{Effect of Blade Twist}

In this study, blade twist is constrained to a range of -22 to 0 degrees, and is investigated to find its effect on induced power and profile power losses in both hovering and forward flight. It is shown in Figure 6 that induced power behaves significantly with respect to blade twist variation in hover and 
forward flight. As Equation (21) shows, the section lift and induced inflow distribution affect induced power loss directly. Figures 7 and 8 depict the section lift time history in hover and forward flight, respectively. When blade twist becomes more negative, the region of the larger section lift moves inboard, its distribution becomes more uniform, and the largest value becomes lower towards the inner portions of blade. This can reduce induced power loss, as shown in Equation (21). Profile power loss is more sensitive with respect to blade twist in forward flight than in hover. As mentioned above, profile power is the bottleneck of rotor performance in forward flight. Figure 9 shows the time history of blade section drag, which determines the profile power loss. There are moderate drag coefficients in the blade tip stations with medium blade twist.
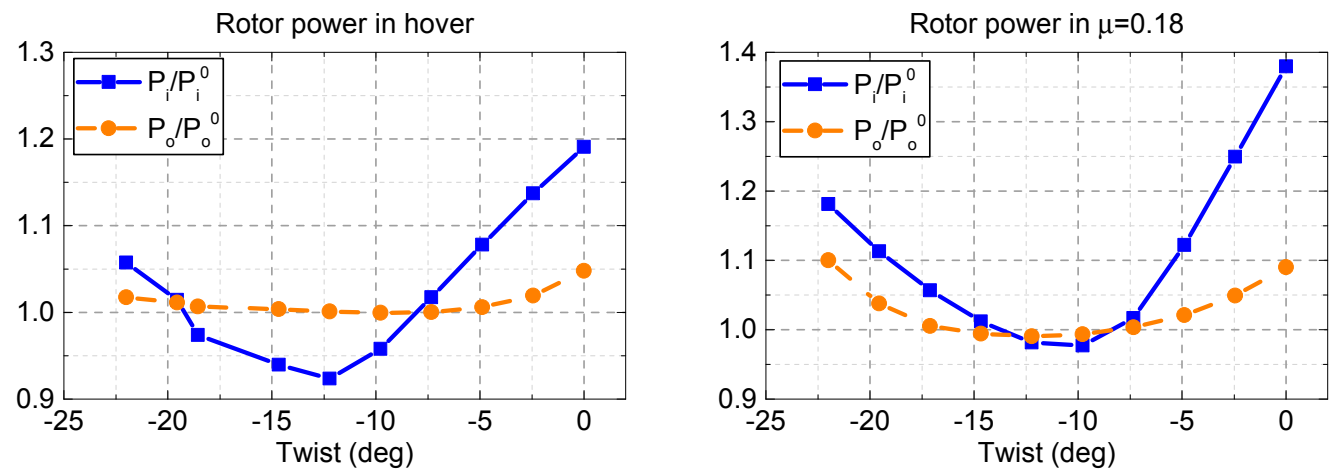

Figure 6. Induced power and profile power with twist variation.

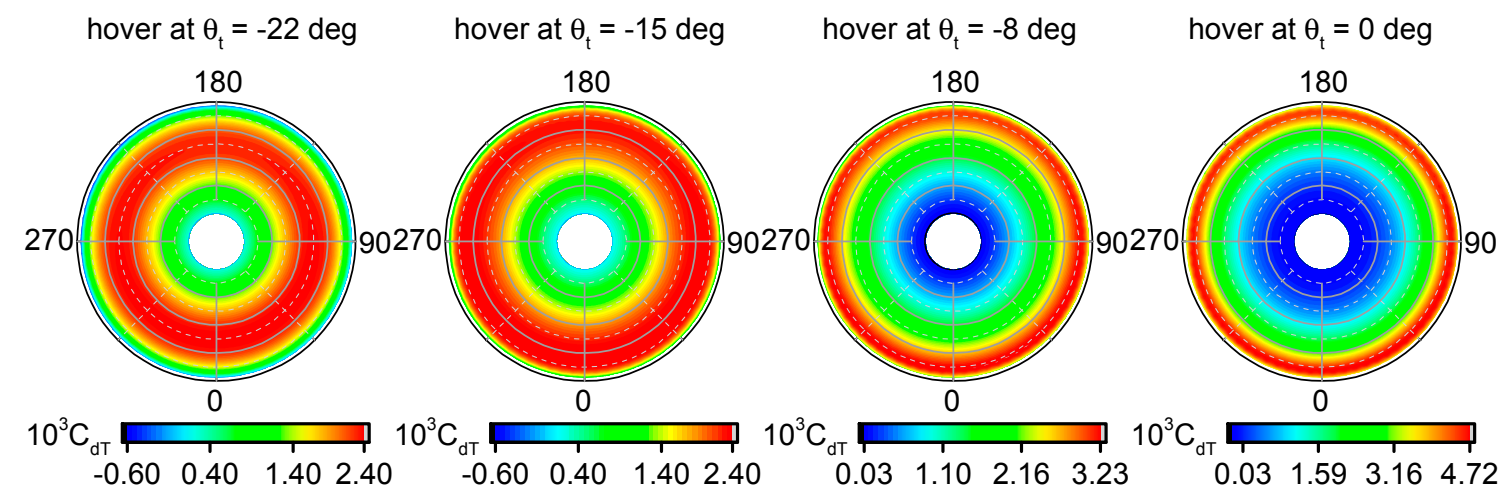

Figure 7. Section lift with twist variations in hover.

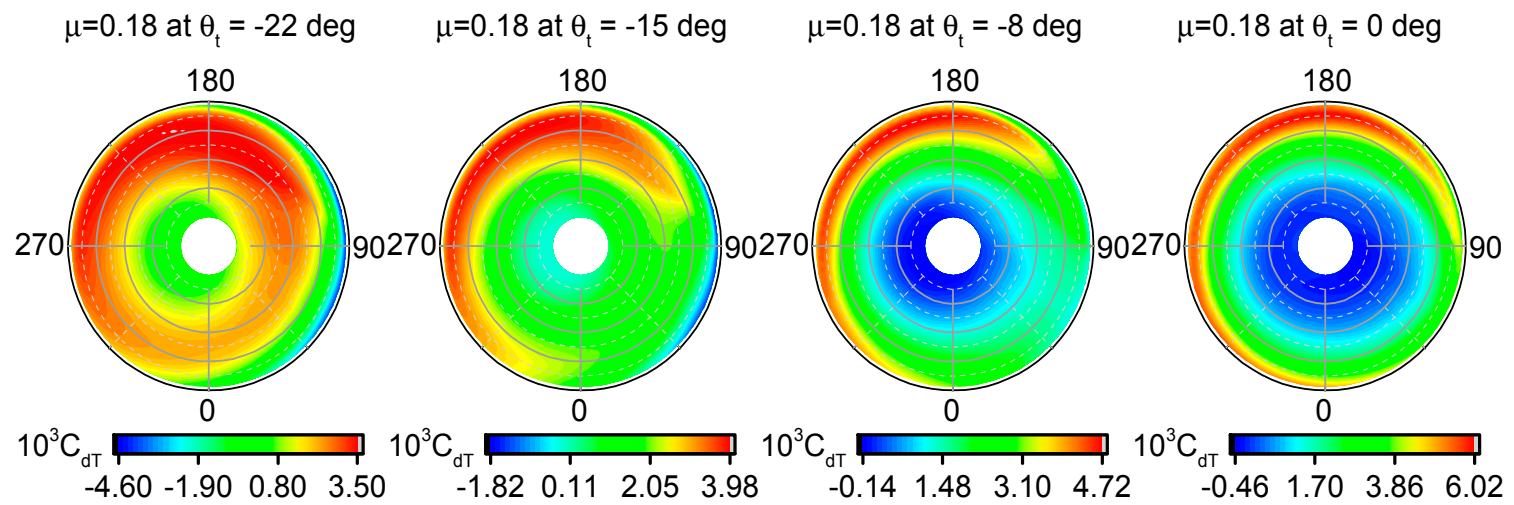

Figure 8. Section lift with twist variations in $\mu=0.18$. 


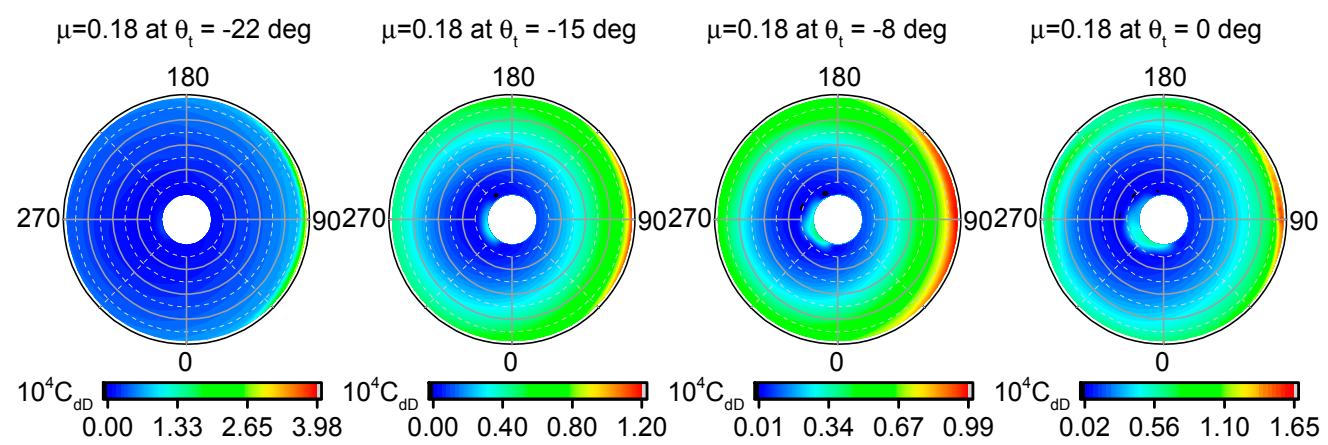

Figure 9. Section drag with twist variations in $\mu=0.18$.

\subsection{Effect of Blade Taper}

Within the framework of actuator disk theory and blade element theory, chord distribution of the optimum hovering rotor is inversely proportional to the radius. However, it is not practical to put this design into production. In general, a linear chord variation is adopted to improve rotor performance, since it is a close approximation of an inversely-proportional rotor over the outer portions of the blade, with little manufacturing cost penalty. The blade taper is defined as a ratio of blade tip chord to root chord in this study. Effects of blade taper on rotor induced power and profile power losses are investigated. The range of taper is confined to between 0.2 and 1 . In order to eliminate the effect of rotor solidity, thrust-weighted solidity is required to be a constant, following Equation (27):

$$
\int_{0}^{1} r^{2} c d r=\text { Const }
$$

In Figure 10, it is shown that the induced power and profile power losses are both affected by blade taper. In both hover and forward flight conditions, profile power loss decreases with taper increases. This is because a shorter chord in the blade tip region can reduce section drag there. Although the chord will elongate near the blade root under the constraint of constant thrust-weighted solidity, the majority of profile power is consumed near the tip, due to the high dynamic pressure. In both hover and forward flight conditions, an increase in blade taper makes lift distribution of blade section more uniform, thus the induced power loss is reduced. In hover, the minimum induced power loss is obtained near 0.4 blade taper.
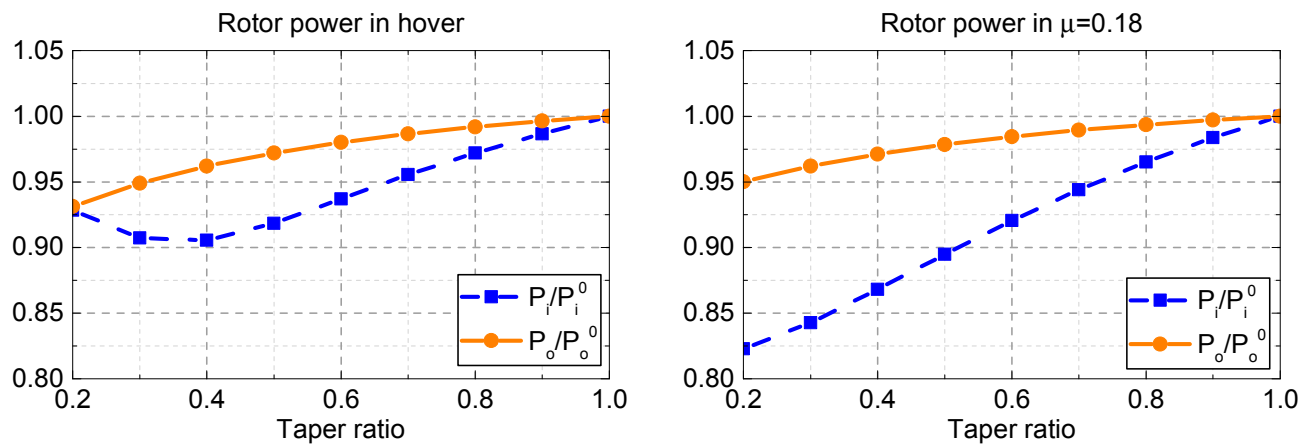

Figure 10. Induced power loss and profile power loss with blade taper variation.

\subsection{Effect of Tip Sweep}

The blade tip plays an important role in rotor aerodynamic performance, since the highest aerodynamic pressure and the strong tip vortex are in this region. A well-designed tip planform can enhance rotor performance significantly. A blade planform with tip sweep is shown in Figure 11. 
The study chooses the tip sweep angle $\Lambda$ and sweep start station $r_{\Lambda}$, where the blade starts to sweep, as design parameters for investigating the effects of tip sweep on rotor performance. It should be noted that the tip sweep may induce the center of aerodynamic pressure to move towards trailing edge. It is possible to intensify the structure coupling between blade pitch and flap in a larger tip sweep. The structure coupling effect is beyond the scope of this study; hence, sweep angle is constrained to within 20 degrees, and its start position is constrained to between 0.85 and 0.95 radius station.

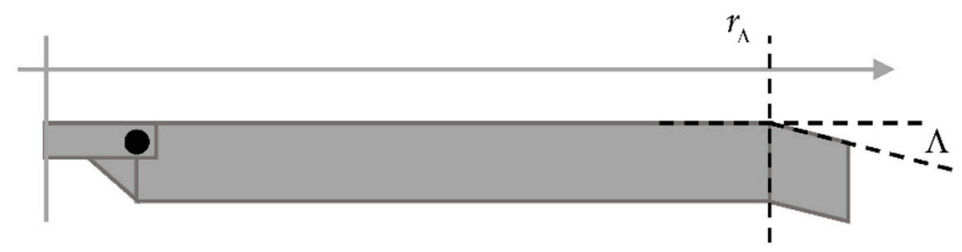

Figure 11. Blade planform.

With a blade tip sweep, the effective chord $\hat{c}$ in the tip region can be expressed as

$$
\hat{c}=c \cos \Lambda
$$

Mach number of flow normal to airfoil leading edge is expressed by

$$
M a_{n}=M a \cos \Lambda
$$

The surface plots of Figure 12 depict the relationship among power loss, sweep angle and sweep start station, in hover and forward flight. It can be seen that in the majority of conditions, the required power decreases with sweep angle increases, both in hover and forward flight. From Equation (28), the sweep angle reduces the effective chord of the blade tip, hence making the blade section lift lower, and its distribution more uniform. The benefit of sweep to profile power is attained by the lower effective Mach number. The lower Mach number normal to the leading edge of the blade section can weaken the compressibility effect, decreasing the drag coefficients in the tip region. It should be noted in Figure 12 that the tip sweep has more influence on rotor performance in forward flight. Thus, a prominent improvement of power loss in forward flight may be attained by a well-designed blade tip.
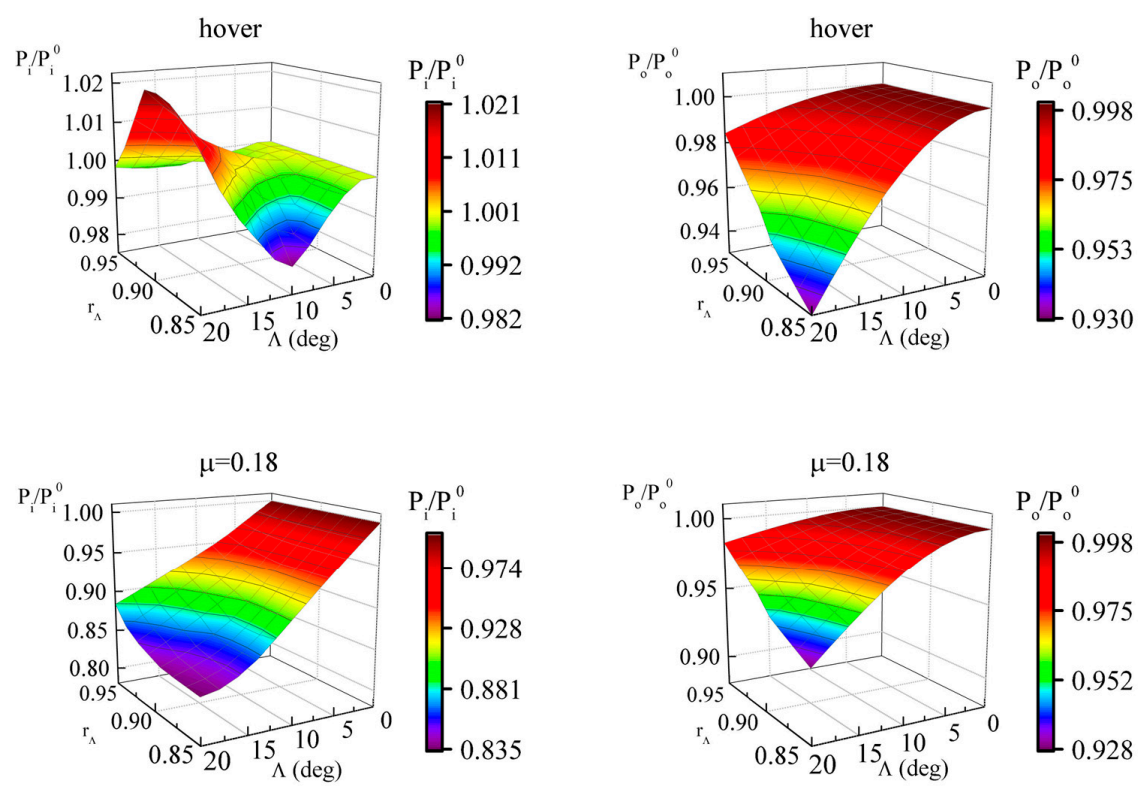

Figure 12. Power losses with tip sweep variations. 


\subsection{Effect of Rotor Speed}

Due to potential structure dynamics problems, most helicopters are constrained to maintain a constant rotor speed in flight. Ranges of 0.65 to 1.15 times the baseline in hover, and 0.7 to 1.15 times baseline in $\mu=0.18$ are assumed in this study. $K_{\omega}$ is defined as the ratio of the new rotor speed to the baseline. Figure 13 shows that profile power loss is more sensitive to rotor speed. In hover condition, profile power loss is reduced with dynamic pressure reduction, which benefits from a reduced rotor speed. In level flight, profile power loss reaches its minimal point at a moderate rotor speed. It is reduced with dynamic pressure reduction on one hand. On the other hand, a higher feathering angle is required in a reduced rotor speed condition to maintain enough lift capacity. Large airfoil drag coefficients may arise, since flow is likely to separate under higher feather conditions. This reflects the fact that profile power improvement is affected by two opposite impacts: decrease in dynamic pressure and increase in attack of angle (AOA). (1) When dynamic pressure reduction is more prominent, profile power loss decreases with rotor speed decreases; (2) when drag coefficients increase due to a higher feathering angle, higher AOA, profile power loss increases dramatically, as Figure 13 shows for a 0.18 advance ratio. In this way, rotor revolution reaches a better point based on freestream velocity and twist.
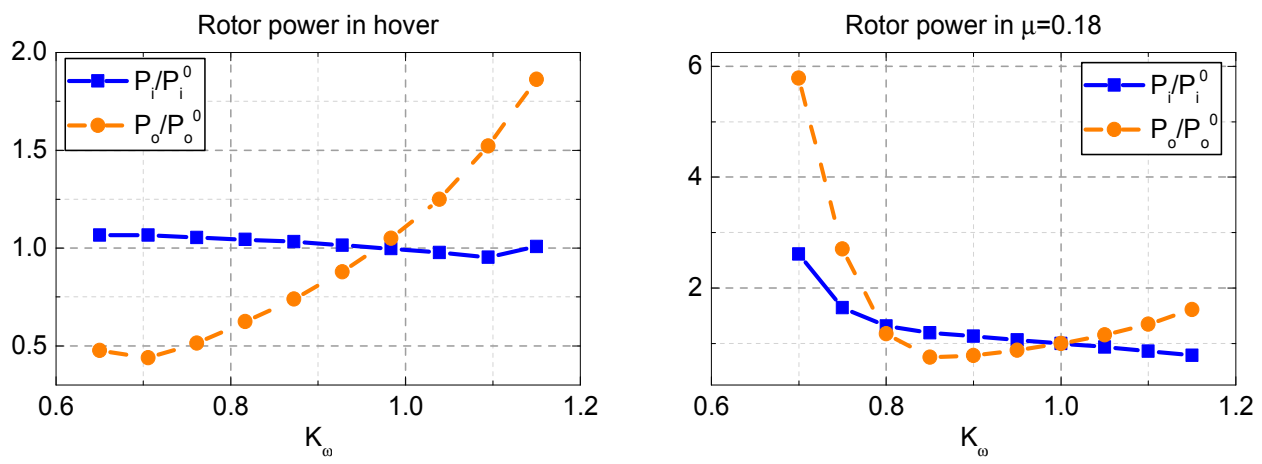

Figure 13. Induced power and profile power with rotor speed variation.

\subsection{Blade Parameter Determination}

The GOE design scheme following Equations (23)-(26) is implemented to find out the optimum rotor performance for an ORS-helicopter. The design variations in this study include blade twist, taper ratio, tip sweep angle, sweep start position and rotor speed. The DPL improvement, with its corresponding blade parameter variations of GOE method, are shown in Figure 14. As mentioned, the DPL is the component that measures rotor performance and design merit directly. Its improvement means a better aerodynamic performance of the rotor itself. The square-point lines express the global optimized solution in overall freestream velocities. In Figure 14a, it can be seen that variable blade technology has a satisfactory improvement for rotor performance in different freestream velocities. The DPL in hover reduces to $83.2 \%$ of the baseline, and it has a better improvement in forward flight, benefitting from the swept tip. The DPL reduces to $74.7 \%$ of the baseline at $124.8 \mathrm{~km} / \mathrm{h}$. The optimum rotor speed is depicted in Figure 14b. In hover and at low freestream velocity, it is allowed a wider range of rotor speed to operate. The consumption of profile power will decrease with a reduced rotor speed. Through a proposed GOE design scheme, the performance improvement, optimum rotor speed, and blade parameters are shown by circle-point lines in Figure 14. It can be seen from Figure 14a that DPL improvement becomes worse than with variable blade technology. Drops of 1.6\%, 1.9\% and 3.0\% are suffered in hover, at moderate freestream velocities like $124.8 \mathrm{~km} / \mathrm{h}$, and at maximum freestream velocities, respectively. Comparing blade parameters by GOE and global optimized solutions, the drops are a consequence of compromises in taper ratio and tip sweep in hover, and taper ratio and blade twist in forward flight. 
Figure 15 depicts the time history plot of DPL by GOE design scheme, with respect to the baseline DPL. It can be seen that the most improvement is attained at the outer portions of the blade, both in hover and in forward flight. That means the parts of power consumed the most is moved towards inner blade portions by the GOE design scheme, including blade twist, taper ratio, tip sweep and rotor speed optimization in this study, making power consumption more homogeneous in the blade.

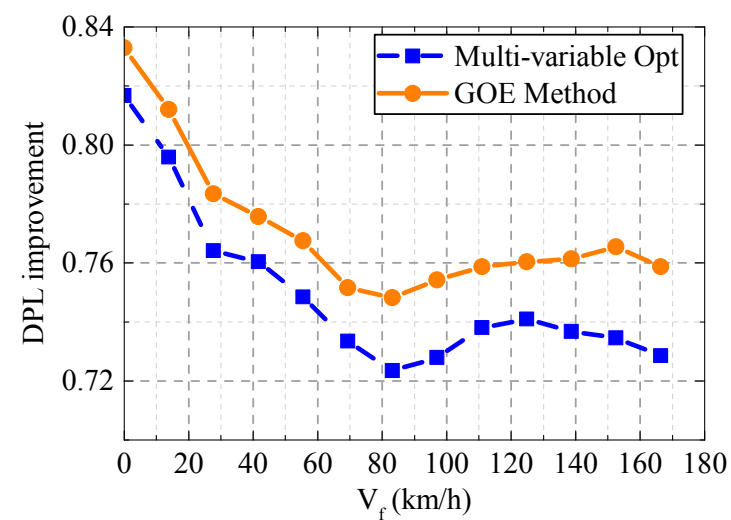

(a)

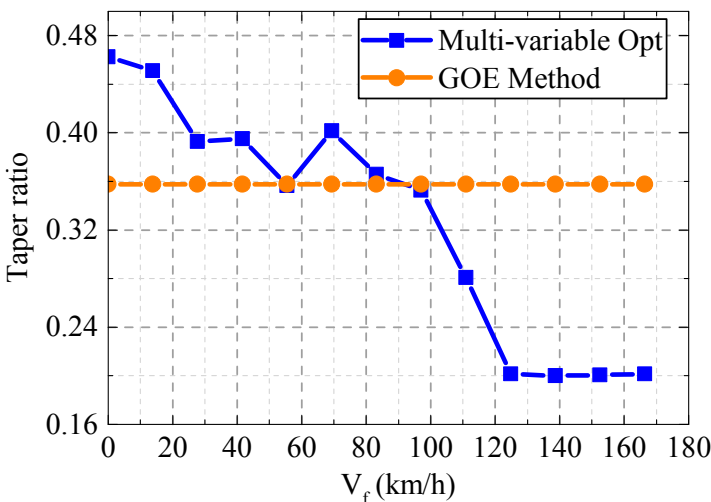

(c)

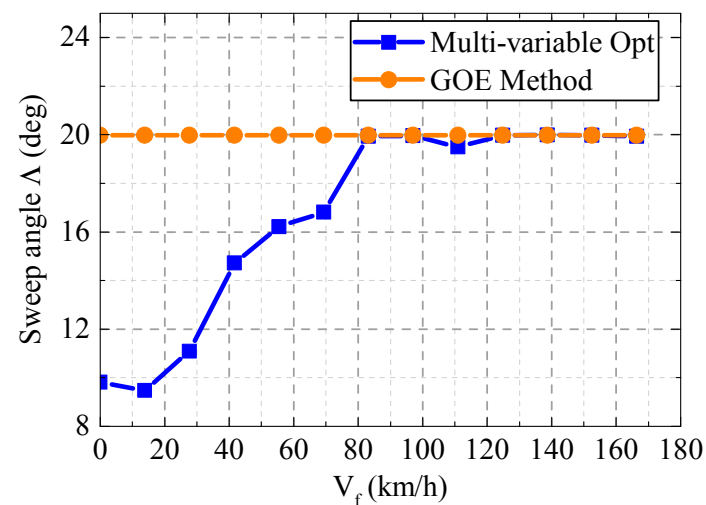

(e)

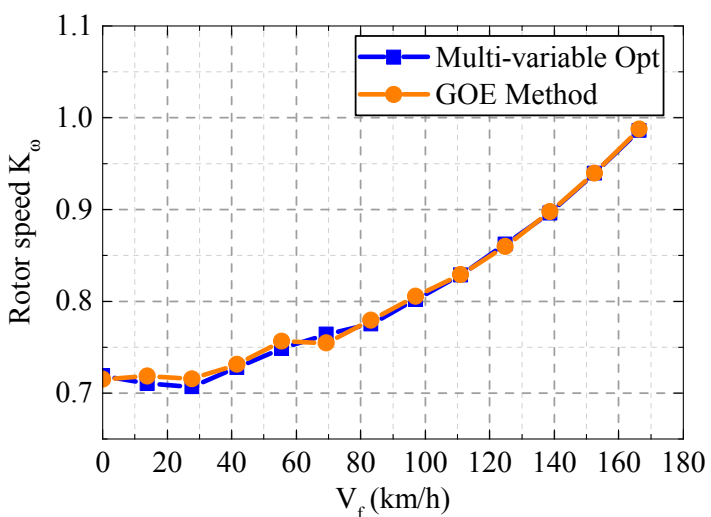

(b)

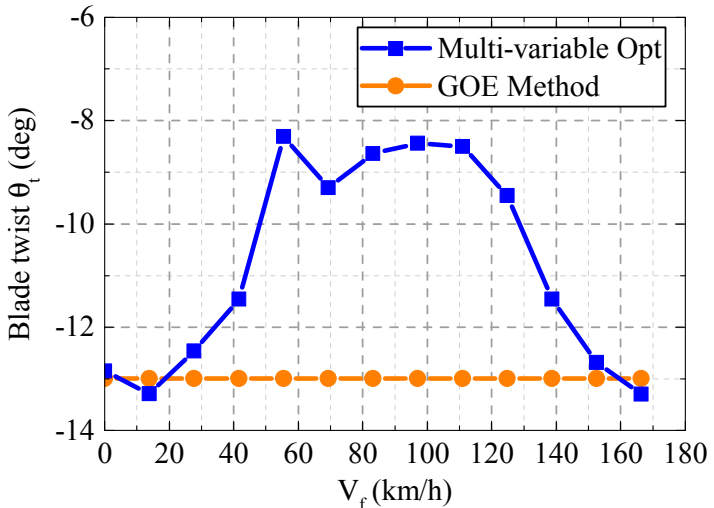

(d)

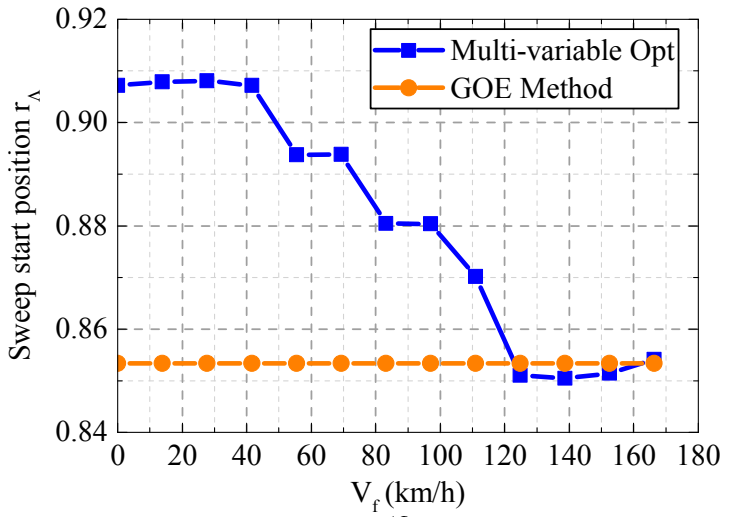

(f)

Figure 14. DPL improvement and its corresponding design parameters in GOE method. (a) DPL improvement; (b) Optimum rotor speed; (c) Taper ratio; (d) Blade twist; (e) Tip sweep angle; (f) Start position of tip sweep. 

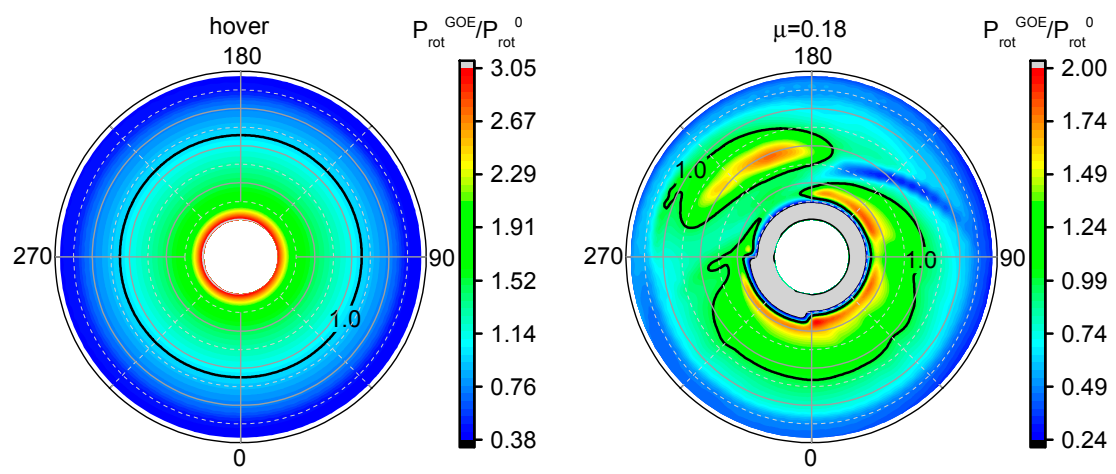

Figure 15. DPL time history by GOE method.

\section{Conclusions}

This study proposes a novel blade optimization method in ORS-helicopters, called GOE, achieving hover and forward flight balance at different freestream velocities. The GOE method integrates with an in-house developed rotorcraft comprehensive code, and is developed to optimize blade twist, taper ratio, tip sweep and rotor speed in ORS-helicopters. The proposed GOE method separates the design procedure into a global optimization step and a secondary optimization step. The global step solves the optimized blade parameters and rotor speed in different freestream velocity conditions, and the secondary step solves the fixed blade parameters and varied rotor speed, with the corresponding objective function closest to the global solution. This study derives the rotor power loss based on blade element theory, and defines DPL as an objective function, which is demonstrated to represent a direct measure of rotor performance. It is also shown to advantageously represent hover and forward flight performances.

This study investigates the effects of blade parameters and rotor speed on induced power and profile power respectively. It reveals that induced power loss is sensitive to blade twist both in hover and forward flight. The region of large blade section lift moves inboard when twist becomes more negative, which makes its distribution close to uniform. A minimal induced power loss with moderate twist is obtained with the synthesized effect of induced inflow and blade section lift. However, profile power dominates in forward flight, and a more twisted blade will induce larger drag coefficients at the blade tip, damaging rotor performance. The blade taper ratio is also investigated in this study. For eliminating the effect of rotor solidity, the study constrains the thrust-weighted solidity to remain unchanged in the GOE scheme. It is concluded that a tapered blade is of benefit to rotor performance. The small tip chord reduces aerodynamic lift and drag near the blade tip. With a slightly elongated chord near the blade root, in accordance with the thrust-weighted solidity condition, only a small portion of profile power loss occurs in the region. It is concluded that significant benefit is attained through the swept back in the blade tip. The sweep angle is limited to within 20 degrees, and the start point is forced to between 0.85 and 0.95 radius station. With an increase in sweep angle, the chord in the blade tip effectively becomes lower. It reduces aerodynamic lift in the tip region and benefits induced power loss in most conditions. Similar characteristics can be observed in sweep start variations. A greater contribution to rotor performance is attained by the tip sweep in forward flight. A reduced Mach number as a result of sweeping the tip backward could weaken the compressibility effect in an advance blade, and decrease drag coefficients and the profile power consumed. The reduction of induced power loss is up to $16.5 \%$, and of profile power loss is up to $7.2 \%$ in $\mu=0.18$, for a blade with 20-degree sweep from a 0.85 radius station.

It is concluded that profile power loss is more sensitive to rotor speed than induced power loss. In hover, about a $56.1 \%$ improvement is achieved in profile power loss at a rotor speed of $70.5 \%$ of baseline. In forward flight in this study, profile power is reduced to $75.3 \%$ at $85 \%$ rotor speed. It is concluded that a variable rotor speed can provide great performance improvement in both hover 
and forward flight. There are two opposite impacts of rotor speed on performance. On one hand, profile power loss becomes lower with dynamic pressure decreases as a result of a reduced rotor speed. On the other hand, a larger drag coefficient arises for a reduced revolution rotor as a consequence of requiring a higher feathering angle to trim. A dramatic rise of profile power loss is encountered in stall. Therefore, the possible rotor speed range becomes narrower in forward flight.

A multivariable optimization is implemented as the first two steps of the GOE method. DPL improves at different freestream velocities, and the minimum improvement is of $18.3 \%$ in hover conditions. It can be concluded variable blade technology is of great significance in improving rotor performance. However, it has so far been difficult to put it into production. The proposed GOE method is implemented for designing a rotor blade for an ORS-helicopter. Through the GOE method, the rotor performance significantly improves. The minimum improvement is by $16.7 \%$ in hover. With an increase in freestream velocity, a rotor designed using the GOE method achieves better improvement as a result of tip sweep design. In cruise flight and maximum flight, the improvement in DPL is about $24 \%$. It is concluded that a rotor designed by the GOE method balances hover and forward flight better.

The proposed GOE design scheme is demonstrated to be effective at determining the optimal blade parameters in an ORS-helicopter, and balance the hover and forward flight performances. We firmly believe the concept of the GOE method could be beneficial to solving the multi-parameter optimization design problems of complex systems. Additionally, there is a relationship among rotor speed, required power and shaft torque. We will extend the GOE method into multi-objective optimization design problems in the future, such as the balance of shaft torque, required power and rotor speed in an ORS-helicopter.

Acknowledgments: The authors would like to thank Haowen Wang from Tsinghua University for valuable discussions on the understanding of rotorcraft dynamics.

Author Contributions: Ming Zhou, Jiayi Xie, Zhifeng Xie and Jun Qiu conceived the research, contributed to literature search; Jiayi Xie and Jun Qiu proposed the GOE method; Jiayi Xie conducted coding and analyzed the data; Jiayi Xie and Jun Qiu wrote and revised the paper.

Conflicts of Interest: The authors declare no conflict of interest.

\section{Notation}

$c$

$C_{T}$

$C_{L}$

$C_{D}$

$C_{p_{i}}$

$C_{P_{0}}$

$d r$

$I_{\beta}$

$K_{\beta}$

$M_{\beta}$

$N_{b}$

$$
P
$$

\author{
airfoil chord \\ rotor thrust coefficient \\ airfoil lift coefficient \\ airfoil drag coefficient \\ induced power coefficient \\ profile power coefficient \\ incremental radius station \\ blade moment of inertia about flap hinge \\ flap hinge restraint \\ blade mass moment about the flap hinge \\ blade number \\ rotor power loss \\ ideal induced power \\ radius station \\ rotor radius
}




$\begin{array}{ll}u_{a} & \text { air velocity } \\ u_{r}, u_{t}, u_{p} & \text { blade section velocity components; radial, tangential } \\ V_{f} & \text { and perpendicular } \\ \alpha_{f} & \text { freestream velocity } \\ \beta_{0}, \beta_{c}, \beta_{s} & \text { pitch angle of hub } \\ \dot{\beta}, \ddot{\beta} & \text { flap coefficients } \\ \varepsilon & \text { time derivative of flap angle, and second derivative } \\ \Gamma & \text { of flap angle } \\ \Gamma_{b} & \text { flap hinge offset } \\ \varphi & \text { trail wake strength } \\ \lambda_{h} & \text { bound circulation } \\ \lambda_{i} & \text { attack of angle with respect to hub plane } \\ \lambda_{i o} & \text { inflow ratio respect to hub plane } \\ \rho & \text { induced velocity ratio } \\ \theta_{0}, \theta_{c}, \theta_{s} & \text { averaged induced velocity ratio } \\ \omega & \text { air density } \\ \mu_{h x}, \mu_{h y}, \mu_{h z} & \text { collective feathering, cosine feathering, sine } \\ \psi & \text { feathering } \\ & \text { rotor speed } \\ & \text { advance ratio in hub } \\ & \text { azimuth angle }\end{array}$

\section{References}

1. Leishman, J.G.; Rosen, K.M. Challenges in the aerodynamic optimization of high-efficiency proprotors. J. Am. Helicopter Soc. 2011, 56. [CrossRef]

2. Le Pape, A. Numerical Aerodynamic Optimization of Helicopter Rotors: Multi-objective Optimization in Hover and Forward Flight Conditions. In Proceedings of the 31st European Rotorcraft Forum, Florence, Italy, 13-15 September 2005.

3. Léon, E.R.; Pape, A.L.; Costes, M.; Désidéri, J.A.; Alfano, D. Concurrent aerodynamic optimization of rotor blades using a nash game method. J. Am. Helicopter Soc. 2016, 61, 1-13. [CrossRef]

4. Imiela, M. High-fidelity optimization framework for helicopter rotors. Aerosp. Sci. Technol. 2012, 23, 2-16. [CrossRef]

5. Leusink, D.; Alfano, D.; Cinnella, P. Multi-fidelity optimization strategy for the industrial aerodynamic design of helicopter rotor blades. Aerosp. Sci. Technol. 2015, 42, 136-147. [CrossRef]

6. Bailly, J.; Ortun, B.; Delrieux, Y. Recent advances in rotor aerodynamic optimization, including structural data update. J. Am. Helicopter Soc. 2017, 62, 1-11. [CrossRef]

7. Khoshlahjeh, M.; Gandhi, F. Helicopter rotor performance improvement with rpm variation and chord extension morphing. In Proceedings of the American Helicopter Society 69th AHS Annual Forum, Arizona, AZ, USA, 21-23 May 2013.

8. Prouty, R. Should we consider variable rotor speeds. Vertiflite 2004, 50, 24-27.

9. Steiner, J.H. An Investigation of Performance Benefits and Trim Requirements of a Variable Speed Helicopter Rotor. Ph.D. Thesis, The Pennsylvania State University, Padova, Italy, 2008.

10. Miste, G. Variable Speed Rotor Helicopters: Optimization of Main Rotor-Turboshaft Engine Integration. Ph.D. Thesis, Università degli Studi di Padova, Padova, Italy, 2015.

11. Guo, W. Flight Control Design for Rotorcraft with Variable Rotor Speed. Ph.D. Thesis, The Pennsylvania State University, State College, PA, USA, 2009.

12. Garavello, A.; Benini, E. Preliminary study on a wide-speed-range helicopter rotor/turboshaft system. J.Aircr. 2012, 49, 1032-1038. [CrossRef]

13. Han, D.; Barakos, G.N. Variable-speed tail rotors for helicopters with variable-speed main rotors. Aeronaut. J. 2017, 121, 433-448. [CrossRef]

14. Ramanujam, R.; Abhishek, A. Performance optimization of variable-speed and variable-geometry rotor concept. J. Aircr. 2017, 54, 476-489. [CrossRef] 
15. Leishman, G.J. Principles of Helicopter Aerodynamics with Cd Extra, 2nd ed.; Cambridge University Press: Cambridge, UK, 2006; pp. 567-654.

16. Drees, J.M. A theory of airflow through rotors and its application to some helicopter problems. J. Helicopter Assoc. G. B. 1949, 3, 79-104.

17. Van der Wall, B.G. Extensions of prescribed wake modelling for helicopter rotor bvi noise investigations. CEAS Aeronaut. J. 2012, 3, 93-115. [CrossRef]

18. Moré, J.J. The Levenberg-Marquardt Algorithm: Implementation and Theory. In Numerical Analysis; Springer: Berlin/Heidelberg, Germany, 1978; pp. 105-116.

19. Ghee, T.A.; Berry, J.D.; Zori, L.A.; Elliott, J.W. Wake Geometry Measurements and Analytical Calculations on a Small-Scale Rotor Model; NASA-TP-3584, L-17449, NAS 1.60:3584, ATCOM-TR-96-A-007; NASA: Hampton, VA, USA, 25 December 1996.

20. Tan, J.F. Research on helicopter Aerodynamic Interaction with Unsteady Panel/Viscous Vortex Particle Hybrid Method. Ph.D. Thesis, Tsinghua University, Beijing, China, 2009.

21. Bhagwat, M. Mathematical Modeling of the Transient Dynamics of Helicopter Rotor Wakes Using a Time-Accurate Free-Vortex Method. Ph.D. Dissertaion, University of Maryland at College Park, Moldova, MD, USA, 2001.

22. Shen, J.; Chopra, I.; Johnson, W. Performance of swashplateless helicopter rotor with trailing-edge flaps for primary flight control. J. Am. Helicopter Soc. 2010, 55. [CrossRef]

(C) 2017 by the authors. Licensee MDPI, Basel, Switzerland. This article is an open access article distributed under the terms and conditions of the Creative Commons Attribution (CC BY) license (http://creativecommons.org/licenses/by/4.0/). 